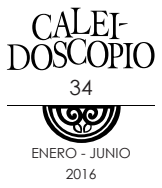

\title{
Perfil sociodemográfico del adolescente que acude a servicios de atención para las adicciones en el estado de Aguascalientes: un estudio exploratorio
}

\section{RESUMEN}

El objetivo de este trabajo fue recabar información sobre el perfil sociodemográfico del adolescente usuario de sustancias que acude a centros de atención primaria en adicciones del estado de Aguascalientes. Se realizó un estudio ex post facto; se revisaron 146 expedientes de julio de 2012 hasta agosto de 2013; se incluyeron adolescentes de entre 12 y 19 años que solicitaron atención. Los resultados indican que los adolescentes consumidores que recibieron tratamiento tienen en promedio 15 años de edad; hombres en su mayoría (68.49\%); e inician el consumo a los 13 años. La sustancia principal de consumo es el alcohol (41.30\% en mujeres y 36\% en hombres), y enseguida sobresale la marihuana ( $26 \%$ en mujeres y $33 \%$ en hombres). Respecto a su contexto familiar y social, la mayoría de los jóvenes tienen familiares consumidores de alguna sustancia adictiva; gran parte de ellos no se encuentra trabajando (77.4\%), y la escolaridad en más del $50 \%$ es de secundaria. Las principales situaciones de consumo son: situaciones que él busca, y en segundo lugar situaciones inespera-

1 Ambas autoras, colaboradoras del proyecto de investigación "Programa de intervención breve para adolescentes que abusan del alcohol y otras drogas: transferencia y reinvención en los Centros Nueva Vida del Estado de Aguascalientes". Departamento de Psicología, Universidad Autónoma de Aguascalientes. margarita_guer@ hotmail.com. 
das. Estos resultados son muy útiles para ampliar la información relevante sobre las características de los adolescentes consumidores de sustancias, de tal manera que se puedan identificar posibles adecuaciones a los tratamientos específicos que son ofrecidos en los centros de atención especializados para esta población, con el fin de aumentar la eficacia en los resultados.

Palabras clave: perfil sociodemográfico, adolescente, atención primaria, consumo de sustancias.

\section{ABSTRACT}

The aim of this article was to request information about sociodemographic teenager profile that seeks addictions attention in prevention centers in the State of Aguascalientes to receive treatment. The study was ex post-facto with a review of 146 cases from July 2012 to August 2013, there were adolescents between 12 and 19 years old who had been on treatment. Results indicate that adolescents have an average of 15 years old; they are mostly men (68.49\%); average of beginning on their consumption is 13 years old. The main drug of abuse is alcohol (41.30\% women, 36\% men) and projecting marijuana (26\% women, $33 \%$ men). Regarding their family and social context, most young people have relatives that are consumers of some addictive substance; many of them are not working, their educational level is in more than $50 \%$ Junior High School. The main consumption situations are the ones they purposely look for, and secondly are the unexpected situations. These results are very useful for expanding relevant information on the characteristics of adolescent users of substance, so that they can identify possible adjustments to specific treatments that are offered at centers of specialized care for this population in order to increase effectiveness results.

Key words: User profile, adolescence, primary care, substance use and abuse.

\section{INTRODUCCIÓN}

Para mejorar y actualizar innovadoras y eficientes estrategias de prevención y tratamiento del consumo de sustancias adictivas que favorezca 
la obtención de resultados más eficaces en las intervenciones, resulta relevante obtener información acerca del perfil sociodemográfico de los adolescentes consumidores de sustancias, y algunos de los factores relacionados a la demanda de tratamiento y el patrón de consumo.

El aumento en el consumo de sustancias en los adolescentes, aunado a la disminución de la edad de inicio de consumo en los últimos años, resulta un problema de salud pública a nivel nacional que debe ser atendido de manera eficaz y efectiva. Resulta necesario desarrollar investigaciones enfocadas a obtener datos sobre características del usuario y del comportamiento del consumo en el adolescente que recibe tratamiento, para poder conocer: edad de inicio, principal droga de consumo, drogas secundarias, tipos de sustancias de consumo, formas de consumo, contexto familiar del usuario consumidor, tipo de tratamiento que solicitan, entre otros datos, que permitan evaluar las condiciones del consumo de sustancias en los adolescentes, así como formular políticas de salud pública enfocadas a la prevención, intervención y rehabilitación de la conducta de consumo. Es por esta razón que surge la inquietud de realizar este estudio exploratorio.

En México existen diferentes fuentes que proveen de información epidemiológica relevante acerca del consumo de sustancias y las distintas poblaciones relacionadas a esta problemática; algunas de éstas son: la Encuesta Nacional de Adicciones (ENA), el Sistema de Información Epidemiológica del Consumo de Drogas (SIECD) de los Centros de Integración Juvenil, Sistema de Vigilancia Epidemiológica de las Adicciones (SISVEA) de la Secretaría de Salud, el Observatorio Mexicano de Alcohol, Tabaco y otras drogas de la Comisión Nacional contra las Adicciones (CONADIC), entre otras. Los resultados de estas fuentes permiten conocer un panorama general del fenómeno del consumo de sustancias a nivel nacional; sin embargo, realizar un acercamiento más específico a nivel estatal y con adolescentes consumidores que acuden a tratamiento, es otra herramienta útil para integrar información más específica de los perfiles sociodemográficos de las diferentes regiones en el país.

Una fuente importante de datos epidemiológicos la integran los resultados y bases de datos de los Centros de Atención para las Adicciones en México, ya que a través de la información de los usuarios que asisten a recibir tratamiento, se pueden conocer características de la población que consume alguna sustancia, y ofrecer alternativas concretas para enfrentar la problemática del consumo, específicamen- 
te de la población adolescente consumidora de alcohol y otras drogas, tomando en cuenta el perfil del adolescente que acude a tratamiento.

Para obtener un perfil sociodemográfico de una población, es necesario comenzar por comprender el tipo de información que se desea identificar. En psicología, el término perfil es entendido como el "conjunto de medidas diferentes de una persona o grupo, cada una de las cuales se expresa en la misma unidad de medición" (Kerlinger, 2002: 185). Por otra parte, se define perfil como aquellas características particulares que proporcionan información sobre una persona o grupo de personas para diferenciarse de otras. Para este estudio, se definirá perfil como: conjunto de características de tipo psicosocial que permite establecer categorías, dentro de las cuales los adolescentes se insertan con la intención de recibir la atención terapéutica pertinente a sus características psicosociales.

La obtención de un perfil de usuario es fundamental como base de la planeación y actualización de los procedimientos que se llevan a cabo para detectar, intervenir y realizar seguimientos en los usuarios, a fin de garantizar la efectividad en los tratamientos implementados. En el servicio de atención en adicciones es fundamental reconocer las características de las personas que buscan tratamiento, el tipo de droga de consumo, la frecuencia, edad, problemáticas asociadas, entre otras, para hacer adaptaciones pertinentes y efectivas para el tratamiento eficaz de los usuarios.

En el caso particular de esta investigación, se desean distinguir características del perfil de usuarios adolescentes que acuden a tratamiento a los centros de atención primaria en adicciones del estado de Aguascalientes. Estos centros tienen el objetivo de: "[ ... ] ofrecer a la comunidad un modelo de intervención temprana contra las adicciones, contemplando desde la prevención del consumo de sustancias psicoactivas y la promoción de la salud mental, hasta el tratamiento breve; ambulatorio, accesible y de calidad" (CONADIC, 2008).

CONADIC (2008) plantea que los objetivos que tienen estos centros de atención para las adicciones son:

1. Prevención universal.

2. Tamizaje, identificación precoz e intervención breve.

3. Tratamiento breve.

4. Derivación oportuna y de calidad. 
5. Prevención de recaídas y cuidados posteriores.

6. Investigación-acción.

En el cuarto objetivo se destaca la necesidad de conocer las características de los usuarios para brindar una atención y tratamiento efectivos, ya sea adaptando las estrategias a las características de los pacientes (edad, género, severidad y curso de la enfermedad, potencia de recaída, necesidad de atención médica, psiquiátrica, legal, entre otras; así como actitud hacia el tratamiento, apoyo familiar y social), y a las características de los servicios (intensidad del servicio, del apoyo social, accesibilidad, variedad profesional, elementos del programa, plan de alta y seguimiento, tasa proporcional personal/paciente), con la finalidad de hacer más eficiente la atención brindada a los usuarios.

$\mathrm{Al}$ respecto, existen diversos estudios que han considerado ciertas características para definir el perfil sociodemográfico del consumidor de sustancias en general. Fantin (2006) mencionó que las sustancias adictivas más consumidas son el alcohol y el tabaco, además que el paciente consumidor presenta baja autoestima, ansiedad e impulsividad.

Por otro lado, Arellanez, Díaz, Wagner y Pérez (2004) definieron las características siguientes de un perfil de usuario consumidor adolescente en la Ciudad de México: a) género masculino, b) expuestos a situaciones y factores estresores, c) consumo de drogas en familiares, d) vulnerabilidad escolar, e) con disponibilidad de sustancias, f) pertenencia a redes sociales disfuncionales, g) uso inadecuado del tiempo libre, h) diversos trastornos de conducta, afectivos y psicológicos, i) dificultad para el manejo de afectos, j) familias con alta inconsistencia en el manejo de la autoridad, límites difusos o rígidos, desagregadas o con pautas cohesivas que dificultan la individuación y débil apoyo.

Es importante saber que la ENA, en su edición de 2011, identificó que 55.2\% de la población comenzó a consumir alcohol a los 17 años o menos (64.9\% de los hombres encuestados y $43.3 \%$ de las mujeres encuestadas); en el caso de drogas ilegales, el promedio de edad de inicio de consumo es de 18.8 ( 16 años en el caso de los hombres y 18 años en el caso de las mujeres encuestadas). Por otra parte, el SISVEA (2009 citado en ENA, 2011) reportó que en 2009 se atendieron 5,437 casos, de los cuales $48.4 \%$ de los hombres eran menores de 18 años y $49 \%$ eran mujeres; por último, informó también que el alcohol es la droga de inicio que más se emplea (49.1\%), tabaco en segundo lugar (18.4\%) y marihuana en tercer lugar (17.8\%). 
El adolescente es influido al consumo por la diversidad de factores de riesgo que presenta, entre los que se encuentran: consumo por parte de familiares, familias desintegradas, depresión, dificultad para el manejo de afectos, tolerancia social ante el consumo, disponibilidad de sustancias, ansiedad, exposición a estrés y uso inadecuado del tiempo libre, riesgo académico, haber sido testigo o parte de una agresión, entre otros (Arellanez et al., 2004; López y Rodríguez-Arias, 2010; Saravia, Gutiérrez y Frech, 2014). Estos factores de riesgo pueden tener como consecuencia el consumo de drogas como forma no adaptativa de afrontamiento.

El consumo de drogas resulta también un recurso para relacionarse socialmente dentro de la cultura juvenil o en sus tiempos de ocio, haciéndolo durante sus reuniones de fines de semana o, en ocasiones, en lugares no habilitados para el consumo, tales como la vía pública, formando así espacios de interacción creados por ellos mismos (Espada et al., 2003; Guzmán-Facundo, Pedrao et al., 2011).

El adolescente que inicia su consumo de alcohol a temprana edad es propenso al consumo posterior de más sustancias adictivas o ilegales (Secades, 1996; Villatoro et al., 2012); si este consumo de alcohol persiste, probablemente se convertirá en un consumo abusivo y de riesgo (Villatoro et al.). Robertson, David y Rao (2004) afirman que los abusadores de alcohol o de tabaco comienzan a consumir marihuana y pueden avanzar con otras drogas sin dejar la de inicio.

Es por lo anterior que la etapa de desarrollo se ve obstaculizada para lograr un crecimiento saludable, debido a la diversidad de consecuencias negativas asociadas a su consumo, como podrían ser: intoxicación etílica, embriaguez, problemas escolares como la deserción, relaciones sexuales no planeadas, accidentes de tráfico, problemas legales, problemas afectivos, ideación suicida, actos antisociales leves o graves, problemas familiares, etc. (Espada et al., 2003; Villatoro et al., 2012).

El cúmulo de información y datos anteriores muestran la necesidad de identificar de manera específica los perfiles de aquellos adolescentes que acuden a un centro de atención para las adicciones a recibir tratamiento de manera temprana. Una de las opciones que se le ofrece al adolescente que ha experimentado, además, consecuencias negativas asociadas a su consumo, es dirigirse a buscar ayuda a centros especializados en el área, que le ofrezcan el cuidado indispensable con base en las necesidades del usuario y el contexto social donde se desarrolla. 
Este tipo de estudios exploratorios son importantes para identificar las características de los adolescentes consumidores de sustancias adictivas y las problemáticas sociales relacionadas a su consumo, con la finalidad de ofrecer tratamientos específicos eficaces para las necesidades y particularidades de los usuarios. Asimismo, es necesario obtener más información respecto a la población de consumidores adolescentes, para contribuir con datos cuantitativos que permitan realizar estrategias de prevención efectivas y cercanas a la realidad sociodemográfica que viven los adolescentes de la comunidad. Por lo anterior, el objetivo de esta investigación fue elaborar un perfil sociodemográfico de los adolescentes consumidores de sustancias que acuden a recibir tratamiento en los centros de atención para las adicciones. Es un estudio de tipo exploratorio y ex post facto para identificar características sociodemográficas, principal droga de consumo, edad de inicio y situaciones de consumo entre los adolescentes.

\section{MÉTODO}

Participantes: la muestra estuvo conformada por un total de 146 expedientes de adolescentes consumidores de alcohol o alguna droga, en el rango de edad de entre 12 y 19 años, que asistieron a recibir tratamiento a uno de cuatro centros de atención para las adicciones del estado de Aguascalientes, atendidos durante el periodo entre julio de 2012 y agosto de 2013. Se revisaron e incluyeron todos los expedientes que cumplieron con los requisitos de inclusión referentes a la edad y motivo de consulta relacionados con el consumo de sustancias.

Escenario: cuatro centros de atención y prevención de adicciones ubicados en los municipios de Aguascalientes, Jesús María, Rincón de Romos y Calvillo, en el estado de Aguascalientes, México.

Tipo de estudio o diseño de investigación: estudio ex post facto, de carácter exploratorio.

Instrumentos y materiales: la revisión de los expedientes de cada centro se realizó con la ayuda de una ficha de identificación que incluía las variables que debía contener un perfil, elaborada para los fines de esta investigación. La ficha contuvo las siguientes variables de estudio: edad, género, población (urbana-rural), escolaridad, empleo, motivo de consulta, droga principal de consumo (la que origina la solicitud del servicio), edad de inicio de consumo, frecuencia de con- 
sumo de droga principal, droga secundaria de consumo, situaciones de consumo, consumo de drogas en familiares (cualquier familiar que viva con el adolescente) y programa de intervención que fue aplicado.

Procedimiento: en un primer momento, se tuvo contacto con el directivo de cada centro de atención para las adicciones, con el propósito de dar a conocer el objetivo de la investigación, así como acordar días y horarios en los cuales se asistiría a los centros para la revisión de los expedientes. En esta entrevista se acordaron los aspectos del manejo de información personal y confidencialidad de los datos.

En un segundo momento, se prosiguió a la revisión de expedientes en cada uno de los centros con la ficha de identificación. Se seleccionaron solamente aquéllos que cumplieran con el requisito de ser usuarios de 12 hasta 19 años de edad, dentro del periodo comprendido entre julio de 2012 y agosto de 2013. La muestra se conformó de 146 expedientes elegidos por conveniencia.

Se realizó una base de datos con ayuda del programa Microsoft Excel para vaciar la información contenida en las fichas. Se codificó cada uno de los datos, y posteriormente se realizaron análisis de estadística descriptiva para poder cumplir el objetivo de la investigación.

\section{RESULTADOS}

\section{Característica de la muestra}

Se logró recabar una muestra de 146 expedientes de usuarios, el rango de edad osciló entre los 12 y 19 años, la edad promedio presentada fue de 15 años. De los adolescentes, $68.49 \%$ fueron hombres y $31.51 \%$ fueron mujeres. Del total de expedientes revisados, $78.08 \%$ pertenece a la población rural (41 expedientes en Jesús María, 40 en Rincón de Romos y 33 en Calvillo) y $21.91 \%$ restante pertenece a la población urbana (30 expedientes en la capital de Aguascalientes y dos expedientes en Jesús María).

De la información obtenida, se encontró que la edad promedio de inicio de consumo es a los 13 años. La edad mínima que presentó un usuario como inicio de consumo fue de 8 años, mientras que la edad máxima fue de 16 años. La droga principal de consumo fue el alcohol, seguida por la marihuana. 
Respecto a la escolaridad de los usuarios, la mayoría está cursando la secundaria $(52.74 \%)$, la preparatoria la estudia $26.71 \%$, y en menor porcentaje tienen como escolaridad la primaria (13.01\%); el restante (7.53\%) no manifestó ninguna de éstas. La mayoría de los adolescentes $(77.4 \%)$ no cuenta con trabajo.

Sobre el consumo por género, en ambos casos el alcohol resulta la droga principal para la mayoría; en las mujeres representa $41.30 \%$, mientras que en los hombres 36\%. La droga en segundo lugar para los hombres es la marihuana en $33 \%$, y para las mujeres, el tabaco en $26.09 \%$. En el caso de los hombres no se encontró ningún caso de consumo de cocaína como droga principal, mientras que en las mujeres se presentó en $4.35 \%$ de los casos. En las mujeres no se identificó ningún caso de poliusuarios, mientras que en los hombres se reportan $6 \%$ (alcohol, marihuana y drogas de diseño), es decir, usuarios de más de dos sustancias adictivas.

De los usuarios, cuya droga principal de consumo es el alcohol, reportan el tabaco como la secundaria; de igual manera, al ser el tabaco la sustancia principal de consumo, la secundaria es el alcohol (en la Gráfica 1 se presenta la relación entre la droga principal de consumo y las secundarias).

En el municipio de Jesús María, Aguascalientes, la principal sustancia de consumo en los adolescentes fue la marihuana en 30\%, seguida de inhalables en $26 \%$, siendo poca la diferencia entre éstos; la frecuencia de consumo en la marihuana, alcohol y tabaco es todos los días, y en el caso de los inhalables mayormente se da el consumo de 2 a 3 veces por semana.

En el municipio de Rincón de Romos, Aguascalientes, se encontró que la droga principal de consumo es el alcohol (40\%), seguida de la marihuana, y su mayor frecuencia de consumo es diariamente en el alcohol, tabaco y marihuana.

En el municipio de Calvillo, Aguascalientes, los adolescentes que asisten a este centro presentaron mayormente consumo de alcohol $(61 \%)$. Las sustancias que presentaron en su mayoría un consumo diario fueron el alcohol y el tabaco.

En el municipio de Aguascalientes se advirtió como droga principal de consumo el alcohol y la marihuana en 33\% cada sustancia; la frecuencia es de dos a tres veces por semana en el caso del alcohol, y diario en el tabaco, marihuana e inhalables. 
El principal motivo de consulta manifestado por los usuarios que acuden a estos centros de atención (sin hacer especificación en algún centro en particular), coincide con las drogas principales de consumo, la más sobresaliente fue el alcohol (28.77\%), seguido de la marihuana (25.34\%), después los inhalables (15.07\%), y en menor medida manifiestan acudir a atención por consecuencias de su consumo (11.64\%), consumo de dos o más sustancias (9.59\%) y consumo de tabaco (8.22\%). El motivo de consulta de menor cantidad fue por cocaína (1.37\%).

Considerando el motivo de consulta, cada centro opta por aplicar ciertos programas de intervención, evaluando también las necesidades de cada uno de los usuarios. El programa que se reportó como el más utilizado por estos centros fue el Programa de Intervención Breve para Adolescentes (PIBA) que inician el consumo de alcohol y otras drogas (Martínez et al., 2009), aunque es importante señalar que hay un porcentaje significativo de casos en los que no se reporta el programa de intervención utilizado.

Tabla 1. Programas de intervención aplicados con adolescentes en los centros de atención para las adicciones.

\begin{tabular}{lc} 
Programa aplicado & Porcentaje \\
\hline No menciona & $41.10 \%$ \\
\hline PIBA & $43.84 \%$ \\
\hline Consejo Breve & $10.96 \%$ \\
\hline Intervención breve para usuarios de marihuana & $1.37 \%$ \\
\hline Valoración psiquiátrica & $1.37 \%$ \\
\hline Bebedores problema & $0.68 \%$ \\
\hline Programa satisfactores cotidianos & $0.68 \%$ \\
\hline
\end{tabular}

En relación con las principales situaciones de consumo, se encontró que la situación primordial de consumo en $60 \%$ de los casos es "situaciones que él mismo busca" -éstas incluyen situaciones donde el adolescente sabe que habrá consumo-; le sigue con 20\% las situaciones inesperadas (ofrecimientos, estar en la calle y encontrarse con amigos que están consumiendo, etc.); en menor cantidad (14\%) están 
las situaciones desagradables (enojos, peleas, problemas familiares, escolares, estar aburrido); y las situaciones agradables (celebraciones en general) con $6 \%$ de ocurrencia.

Finalmente, dentro de la búsqueda se encontró que $52.74 \%$ de los adolescentes usuarios tienen familiares consumidores, y en la mayoría de los casos es el papá.

\section{DISCUSIÓN}

El propósito de este estudio exploratorio consistió en identificar un primer perfil del usuario adolescente que acude a centros de atención primaria en adicciones en el estado de Aguascalientes. Para abordar el objetivo se construyó una ficha de identificación basada en las necesidades de la investigación; se realizó el trabajo de campo y se obtuvieron los resultados descritos en cuanto a las características indagadas. Se logró revisar y analizar 146 expedientes con información similar por cada centro; no todos los expedientes contenían la misma información, pero se hizo un esfuerzo por unificar los datos encontrados.

De la información recabada en los expedientes revisados, se concluye que el perfil del adolescente, bajo los criterios de la ficha de identificación empleada, es el siguiente: los adolescentes hidrocálidos usuarios de sustancias comienzan a consumir en promedio a los 13 años, sin embargo, es hasta los 15 años aproximadamente cuando asisten a solicitar algún tratamiento a los centros de atención primaria para las adicciones. Esta información resulta relevante, ya que el tiempo que transcurre entre el inicio del consumo y la búsqueda de ayuda para modificarlo es alrededor de dos años.

Siguiendo con los resultados para el desarrollo del perfil sociodemográfico, se encontró que la droga principal de consumo es el alcohol, seguida de la marihuana con una frecuencia diaria de consumo. Respecto a la droga secundaria, los datos obtenidos coinciden con lo observado en otros estudios, en los que afirman que los que presentan abuso temprano de la sustancia de inicio del consumo son más propensos a consumir una segunda y hasta una tercera droga (Secades, 1996; Robertson, David y Rao, 2004).

Estos resultados coinciden con los afirmados por Fantin (2006), en lo que concierne al consumo de alcohol como droga principal, pero en el caso de los adolescentes del estado de Aguascalientes, el tabaco no 
es la segunda droga principal, sino la marihuana; este tipo de hallazgos nos permiten conocer las especificidades en la población con la que se trabaja y, determinar que existe la necesidad de incrementar el trabajo preventivo para el no consumo de marihuana, ya que comienza a emplearse de manera más regular en la población.

El PIBA (Martínez et al., 2004; 2009) es el más utilizado dentro de los centros para el tratamiento de esta etapa de desarrollo, con $43.84 \%$ de los casos.

Dentro de su manual, el PIBA señala lo siguiente: "El Programa de Intervención Breve para Adolescentes está diseñado para implementarse dentro de las escuelas de nivel medio y medio superior; sin embargo, también puede aplicarse en instituciones de salud dedicadas a la prevención y atención de las adicciones" (Martínez et al., 2009). Con base en los resultados y tomando en cuenta que el adolescente tarda alrededor de dos años en buscar ayuda respecto a su consumo, y además presentando varias sustancias de consumo, se propone que se realicen esfuerzos a fin de atender a los adolescentes dentro de sus instituciones escolares para una detección más temprana y efectiva.

La principal situación de consumo ocurre en lugares en los que el adolescente asiste con la certeza de que habrá consumo (situaciones que él busca); este consumo, de acuerdo con los datos encontrados, se presenta ya sea con algún familiar o con el grupo de pares. Sin duda, el que exista consumo en la familia es un factor de riesgo para el inicio y abuso en el adolescente. Ruiz et al. (2014) afirmaron que cuando existe el consumo de sustancias en la familia, muy probablemente el hijo adolescente consumirá en ese momento o en un momento posterior. En el caso de los adolescentes que acuden al centro de tratamiento, la mayoría reportan consumo de familiares, siendo en la mayoría de los casos el padre.

Es de relevancia que dentro de los resultados obtenidos, casi en $50 \%$ de los expedientes revisados no se especifica si el adolescente finalizó el tratamiento o desertó durante alguna de las etapas del mismo. Con base en esto, sería interesante analizar, en el caso de que no hayan concluido su tratamiento, ¿cuál fue el motivo de esto?, así como conocer otros aspectos del usuario como: interés en asistir al tratamiento, cuántos terminaron un tratamiento completo, consecuencias del consumo, tener información sobre la pertinencia o no de hacer mejoras a los programas utilizados o la realización de nuevos programas en los centros que atiendan las necesidades actuales en el tema de adicciones. 
Las principales aportaciones de este estudio radican en que es un primer acercamiento a la población adolescente que acude a recibir tratamiento sobre el consumo de sustancias, a diferencia de otras investigaciones que aportan resultados referentes a los adolescentes, pero en población general, sin identificar si acuden a recibir alguna intervención. Además, es importante señalar que la población utilizada para obtener estos datos es población clínica, adolescentes que están presentando problemas de consumo.

Éste fue un estudio exploratorio, y posteriormente se realizará una ampliación de la muestra para poder establecer un perfil sociodemográfico más completo de los usuarios adolescentes que acuden a los centros de atención para las adicciones, así como para definir su patrón de consumo; de esta manera, podemos ofrecer datos con evidencia empírica a fin de evaluar la eficacia y utilidad de los servicios e intervenciones que ofrecen estos centros a los usuarios.

Como limitantes se detalla que la cantidad de expedientes revisados y los datos faltantes en cada uno de ellos, no permitió establecer categorías de perfiles que nos posibilitaran identificar los diferentes tipos de adolescentes que acuden a estos centros en busca de ayuda, como se menciona en el párrafo anterior, se planea posteriormente aumentar la muestra para poder realizar estos análisis.

Finalmente, se reafirma la importancia de hacer más investigaciones sobre los perfiles que tienen los usuarios de drogas en general, y propiamente indagar sobre las características de los adolescentes consumidores, para conocer sus necesidades específicas, las cuales nos aportarán información relevante para el desarrollo y actualización de las intervenciones sobre el tipo de sustancias específicas y la población consumidora, adaptadas a su perfil sociodemográfico.

\section{REFERENCIAS}

Arellanez, H. J., Díaz N. D., Wagner E. F. y Pérez I. V. (2004). Factores psicosociales asociados con el abuso y la dependencia de drogas entre adolescentes: análisis bivariados de un estudio de casos y controles. Salud Mental, 27(3), 54-64. Recuperado de: http://www.inprf-cd. org.mx/pdf/sm2703/sm270354ZNN.pdf.

Comisión Nacional contra las Adicciones. (2008). Centros de Atención Primaria en Adicciones. Centros "Nueva Vida". Secretaría de Salud: México. 
Encuesta Nacional de Adicciones 2011. (2012). Instituto Nacional de Psiquiatría Ramón de la Fuente Muñíz [INPRFM] / Secretaría de Salud. México.

Espada, J. P., Méndez, X., Griffin, K. W. y Botvin, G. J. (2003). Adolescencia: consumo de alcohol y otras drogas. Papeles del Psicólogo, 84, 9-17. Recuperado de: http://www.redalyc.org/pdf/778/77808402.pdf.

Fantin, M. (2006). Perfil de personalidad y consumo de drogas en adolescentes escolarizados. Adicciones, 18(3), 285-292.

Guzmán-Facundo, F., Pedrao, L., López-García, K., Alonso-Castillo, M. y Esparza-Almanza, S. (2011). El consumo de drogas como una práctica cultural dentro de las pandillas. Revista Lastino-Am. Enfermagem, 19, 839-847.

Instituto Nacional de Psiquiatría Ramón de la Fuente Muñiz. (2011). Encuesta Nacional de Adicciones 2011: reporte de drogas.

Kerlinger, F. N. (2002). Investigación del comportamiento. México: McGraw Hill.

López, S. y Rodríguez-Arias, J. (2010). Factores de riesgo y de protección en el consumo de drogas en adolescentes y diferencias según edad y sexo. Psicothema, 22(4), 568-573.

Martínez, K. I., Salazar, M. L., Ruiz, G. M., Barrientos, V. y Ayala, H. E. (2004). Programa de Intervención Breve para Adolescentes que inician el consumo de alcohol y otras drogas. Manual del terapeuta. México: Universidad Nacional Autónoma de México.

Martínez, K. I., Salazar, M. L., Ruiz, G. M., Barrientos, V. y Ayala, H. E. (2009). Programa de Intervención Breve para Adolescentes que inician el consumo de alcohol y otras drogas. Manual del terapeuta Segunda Edición. México: Universidad Nacional Autónoma de México.

Robertson, E. B., David, S. L., y Rao, S. A. (2004). Preventing drug use among children and adolescents. A research based guide for parents, educators and community leaders. Maryland: National Institute on Drug Abuse (NIDA).

Ruiz, H., Herrera, A., Martínez, A., Supervielle, M. (2014). Comportamiento adictivo de la familia como factor de riesgo de consumo de drogas en jóvenes y adolescentes adictos. Revista Cubana de Investigaciones Biomédicas. 33(4), 402-409.

Saravia, J., Gutiérrez, C. y Frech, H. (2014). Factores asociados al inicio de consumo de drogas ilícitas en adolescentes de educación secundaria. Revista Peruana de Epidemiología, 18(1), 1-7.

Secades, R. (1996). Alcoholismo juvenil. Prevención y tratamiento. Madrid: Pirámide. 
Secretario Técnico del Consejo Nacional contra las Adicciones. (2008). Modelo de atención UNEME-CAPA. Centros de Atención Primaria en Adicciones "Centros Nueva Vida". Recuperado de: www.conadic.gob.mx.

Sistema de Vigilancia Epidemiológica de las Adicciones 2011. (2013). Dirección General de Epidemiología. México: Secretaría de Salud.

Villatoro, J., Bustos, M., Oliva, N., Moreno, L., Gaytán, F., Fregoso, D., Chávez, J., Martín del Campo, R., Medina-Mora, M. y Gutiérrez, M. L. (2012). ¿Es el alcohol una problemática aislada en los y las adolescentes? En Actualidades en adicciones 2012: consumo de alcohol y tabaco en México. México: CONADIC.

\section{ANEXos}

GRÁFICA 1. Drogas secundarias de consumo en relación con la droga principal.

Droga principal y secundaria

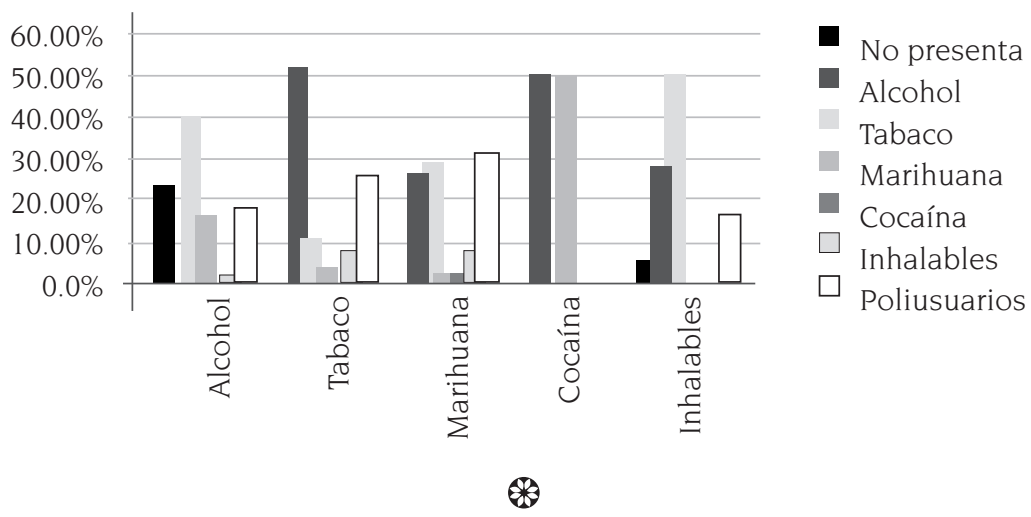

